\title{
Simultaneous optically sectioned fluorescence and optical coherence microscopy with full-field illumination
}

\author{
Houssine Makhlouf, ${ }^{1}$ Karen Perronet, ${ }^{1}$ Guillaume Dupuis, ${ }^{2}$ Sandrine Lévêque-Fort, ${ }^{2}$ and Arnaud Dubois ${ }^{1, *}$ \\ ${ }^{1}$ Laboratoire Charles Fabry, CNRS UMR 8501, Institut d'Optique Graduate School, University Paris-Sud, \\ 2 Avenue Augustin Fresnel, 91127 Palaiseau Cedex, France \\ ${ }^{2}$ Centre de Photonique Biomédicale (CLUPS)_Institut des Sciences Moléculaires d'Orsay, University Paris-Sud, 91405 Orsay, France \\ *Corresponding author: arnaud.dubois@institutoptique.fr
}

Received January 23, 2012; accepted March 1, 2012; posted March 19, 2012 (Doc. ID 161946); published May 7, 2012

\begin{abstract}
Full-field optical coherence microscopy (FF-OCM) and optically sectioned fluorescence microscopy are two imaging techniques that are implemented here in a novel dual modality instrument. The two imaging modalities use a broad field illumination to acquire the entire field of view without raster scanning. Optical sectioning is achieved in both imaging modalities owing to the coherence gating property of light for FF-OCM, and a structured illumination setup for fluorescence microscopy. Complementary image data are provided by the dual modality instrument in the context of biological tissue screening. FF-OCM imaging modality shows the tissue microarchitecture, while fluorescence microscopy highlights specific tissue features with cellular-level resolution by using targeting contrast agents. Complementary tissue morphology and biochemical features could potentially improve the understanding of cellular functions and disease diagnosis. (c) 2012 Optical Society of America

OCIS codes: $\quad 170.4500,170.2520,170.2945,180.3170$.
\end{abstract}

Optical coherence tomography (OCT) is a scanning imaging technique based on the detection of interference between light backscattered by a sample and reference light. It is used in biomedical applications to image the architecture of biological specimen with micrometerscale resolution [1]. The scanning setup of OCT can be implemented so that cross-sectional or en face images are collected. In the latter case, the technique is specifically referred to as optical coherence microscopy (OCM) [2]. Fluorescence microscopy is another imaging technique that provides spatially resolved information on the biochemical properties of tissues using endogenous [3] or exogenous fluorophores [4]. OCT and OCM have been combined with fluorescence microscopy to yield complementary morphological and functional information $[5,6]$. However, the scanning nature of standard OCT and OCM systems does not allow a full integration with conventional wide-field fluorescence microscopy. In this Letter, we report on a hybrid OCM and fluorescence imaging system using an alternative approach to standard OCM, that is, full-field (FF)-OCM [7]. In FF-OCM, the entire field of view is illuminated and an en face image is reconstructed without any scanning. The principle of whole field imaging is common to both FF-OCM and fluorescence imaging techniques so that it is relevant to combine both into a single instrument.

The layout of the dual modality imaging system is shown in Fig. 1. The FF-OCM imaging modality is based on the system reported in [] ]. A halogen lamp is used as the low-coherence light source. It is set up in a Kohler illumination arrangement to achieve uniform illumination of the sample. The source filament is imaged onto an aperture stop by a collector lens. Lens L3 $(f=$ $150 \mathrm{~mm}$ ) relays this image onto the back focal plane of $20 \times / 0.5$ NA water-immersion microscope objectives MO (Olympus). A 50/50 beam splitter separates the light beam into a reference arm and a sample arm of a Linnik interferometer with identical microscope objectives in each arm. A reference mirror ( $2 \%$ reflectivity) and a sample are placed in the focal planes of the microscope objectives. The length of the reference arm is adjusted by a motorized translation stage to set the FF-OCM imaging plane within the sample. The microscope objective in the sample arm is mounted on another motorized translation stage and is moved appropriately to focus on the imaging plane. The reflected reference light and the backscattered light from the sample combine at the output of the interferometer. Infrared light $(\lambda>650 \mathrm{~nm})$ is

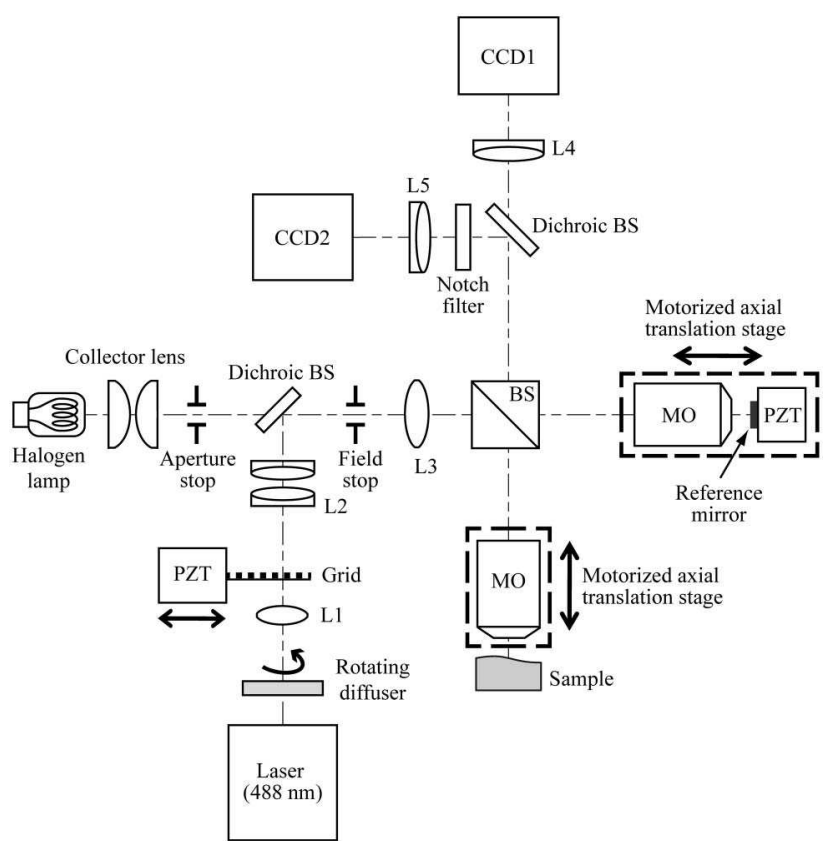

Fig. 1. Layout of the dual modality imaging system: BS, beam splitter; MO, microscope objective; PZT, piezoelectric transducer; L1/L3, singlet lenses; L2, pair of achromatic doublets; L4/L5, achromatic doublets. 
transmitted by a dichroic beam splitter and focused by achromatic doublet lens L4 $(f=300 \mathrm{~mm})$ onto a twodimensional (2D) detector CCD1 (Dalsa, Dalstar 1M15, $1024 \times 1024$ pixels, $15 \mathrm{fps}$ ). An interferogram is formed on the detector plane, which consists of the combined image of the in-focus sample plane and reference mirror. The reference mirror is moved axially by a piezoelectric transducer (PZT). Multiple phase-shifted interferograms are acquired for various positions of the reference mirror. A mathematical combination of the acquired interferograms allows reconstruction of an en face image of the sample at the focusing depth. An algorithm based on the acquisition of two phase-shifted interferometric images was used to reconstruct the image results showed in this report [8].

A $488 \mathrm{~nm}$ solid-state laser source (Coherent, 488-200 CRDM) is used in the fluorescence imaging modality. The laser beam is coupled into the system by a dichroic beam splitter that reflects visible light $(\lambda<700 \mathrm{~nm})$ and transmits infrared light $(\lambda>700 \mathrm{~nm})$. A spinning ground glass plate (rotating diffuser) is placed in the path of the laser beam to suppress the effect of speckle. Lens L1 $(f=100 \mathrm{~mm})$ is used to expand the size of the illumination field on the sample. The optical subsystem consisting of lenses L1, L2, and L3 images the surface of the ground glass plate onto the back focal plane of microscope objectives MO, so that it is not visible in the resulting image. Prior to imaging, the sample is stained with a targeting fluorophore. Visible fluorescent light $(\lambda<650 \mathrm{~nm})$ emitted by the sample is reflected by the dichroic beam splitter and focused onto $2 \mathrm{D}$ detector CCD2 (Photometrics, CoolSnap HQ, $1392 \times 1040$ pixels, $10 \mathrm{fps})$ by achromatic doublet lens L5 $(f=300 \mathrm{~mm})$. A notch filter (Semrock, NF-488) is used to suppress the excitation light. A 20 line pairs/mm grid is placed in the laser beam path after L1 and is imaged by L2 on the field stop plane that is conjugate of the sample plane. L2 consists of a pair of achromatic doublets $(f=60 \mathrm{~mm}$ for each) achieving 1:1 imaging. The grid is mounted on a PZT that controls its transverse positioning. Images of the sample are acquired for three positions of the grid. These positions are shifted by one third of the grid period with respect to each other. The collected images $I_{1}, I_{2}$, and $I_{3}$ are processed to get rid of the grid pattern and compute the optically sectioned image of the sample [9]:

$$
I_{s}=\sqrt{\left(I_{1}-I_{2}\right)^{2}+\left(I_{1}-I_{3}\right)^{2}+\left(I_{2}-I_{3}\right)^{2}} .
$$

This is the so-called structured illumination microscopy (SIM) technique. The conventional wide-field fluorescence image is obtained from the algebraic summation of the three raw acquired images.

The images obtained from the FF-OCM imaging modality and the optically sectioned fluorescence imaging modality can be displayed separately or overlaid to show different information. The detectors used in the two imaging modalities have different formats in terms of pixel number and pixel size. To overlay the fluorescence and FF-OCM images, it was necessary to crop and then interpolate the initial FF-OCM image so that it shows the same field of view and has the same number of data points as the fluorescence image.
The coherence function associated with the halogen lamp source was determined experimentally. Its FWHM gives the axial resolution of the FF-OCM imaging modality and was measured to be $1.5 \mu \mathrm{m}$ in air $(1.1 \mu \mathrm{m}$ in tissue assuming a refractive index of 1.4). The theoretical lateral resolution is $0.9 \mu \mathrm{m}$ at $800 \mathrm{~nm}$ center wavelength. A typical number of $10 \mathrm{FF}-\mathrm{OCM}$ images are acquired and averaged to increase the dynamic range. The detection sensitivity was measured to be $89 \mathrm{~dB}$. The fluorescence point spread function was measured by imaging fluorescent beads. With $0.5 \mu \mathrm{m}$ diameter beads, a lateral resolution of $0.7 \mu \mathrm{m}$ was determined, which is comparable to the theoretical value of $0.6 \mu \mathrm{m}$ at a wavelength of $520 \mathrm{~nm}$. Z-stack images of $1 \mu \mathrm{m}$ diameter beads were acquired. The axial resolution was measured to be $6.0 \mu \mathrm{m}$. A $4.5 \mu \mathrm{m}$ theoretical axial resolution was computed based on the axial response function derived in [9]. The discrepancy between measured and theoretical values is attributed to the presence of optical aberration in the imaging system, including the aberration associated with the imaging of the grid into the sample. Simultaneous acquisition of a fluorescence image and an FF-OCM image takes $2.7 \mathrm{~s}$.

Samples of excised mouse tissues were topically stained with Acridine Orange (AO) and set between a glass slide and a coverslip. (The chromatic dispersion introduced by the coverslip was compensated by placing another coverslip in the reference arm.) AO is a nucleic acid stain with dual peak emission wavelengths around $520 \mathrm{~nm}$ and $650 \mathrm{~nm}$ when bound to DNA and RNA, respectively. Imaging results obtained with tongue and colon are shown in Figs. $\underline{2}$ and $\underline{3}$, respectively. Figures 2 (a) and $2(\mathrm{~b})$ show the conventional and the optically sectioned fluorescence images, respectively. Most out-offocus light is rejected in the optically sectioned image. The general structure of the tongue sample is revealed by the FF-OCM image shown in Fig. 2(c). The optically sectioned fluorescence image and the FF-OCM image are colored in shades of green and red, respectively, and overlaid to form Fig. 2(d) for direct comparison.
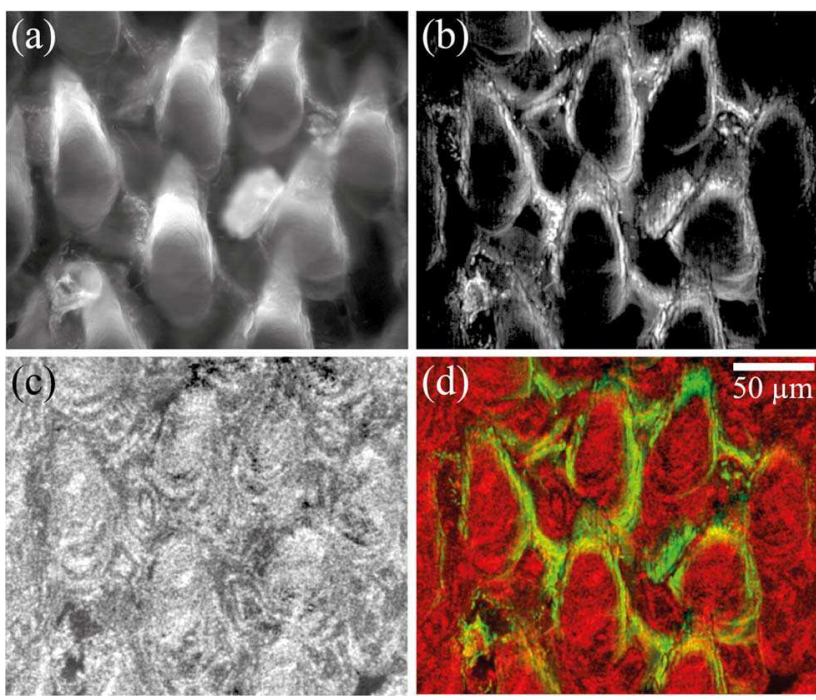

Fig. 2. (Color online) (a) Conventional fluorescence image, (b) optically sectioned fluorescence image, and (c) FF-OCM image of mouse colon stained with AO. (d) Superposition of (b) and (c) in pseudocolors. 

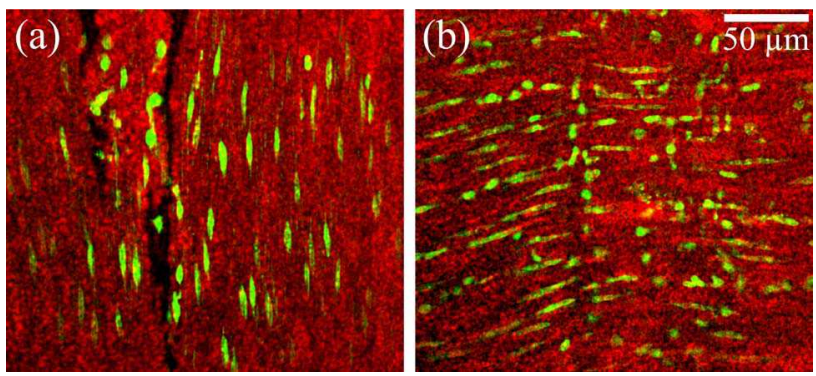

Fig. 3. (Color online) Combined optically sectioned fluorescence image and FF-OCM image in pseudocolors of mouse colon stained with AO. Sample shown at depths of (a) 20 and (b) $40 \mu \mathrm{m}$ below the surface.

The fluorescent nucleic structures delineate the edge of the papillae within the tongue microarchitecture. The imaging depth was about $20 \mu \mathrm{m}$ below the tissue surface. Figure 3 shows the pseudocolored overlay of the optically sectioned fluorescence image and the FF-OCM image of the colon sample at different imaging depths. Figures $3(\mathrm{a})$ and $3(\mathrm{~b})$ correspond to sample planes that are located about 20 and $40 \mu \mathrm{m}$ below the surface, respectively. The fluorescence information reveals individual cell nuclei, while the FF-OCM data show a fibrous pattern that could be connective tissue. The two imaging planes contain different structures that are oriented perpendicularly with respect to each other.

The fluorescence and FF-OCM images show different morphological information. The fluorescence information could also be associated with biochemical processes at the cellular level. An integrated imaging system capable of optically sectioned fluorescence imaging and FF-OCM imaging could potentially be valuable in biomedical applications by providing complementary information during biological tissue screening. This has the potential for better understanding cellular functions and improving the ability to detect pathologies by monitoring morphological and biochemical changes in tissues. The reported imaging system could be used in conjunction with a biopsy procedure. Excised tissue samples could be explored with minimal preparation as opposed to standard histopathology process.

The combined fluorescence and FF-OCM imaging system is novel in that the entire field of view is imaged without raster scanning. Moreover, the fluorescence imaging modality of the reported combined system achieves optical sectioning by using a SIM technique. The system is flexible in that other target-specific fluorophores can be used, provided that they emit in the range of wavelengths below $650 \mathrm{~nm}$ and the appropriate excitation light source is used. Microscope objectives with higher NA can also be used to improve the lateral resolution in both modalities and the axial resolution of the fluorescence SIM technique. Simultaneous acquisition enables capturing an image of the same tissue location regardless of sample motion. However, this motion needs to be negligible during the acquisition time to avoid image blurring. Overlay- ing the images obtained from each imaging modality highlights their differences.

The imaging depth is limited by tissue scattering and absorption. The fluorescence modality dictates the maximum imaging depth while being able to coregister a fluorescence image and an FF-OCM image at the same plane location in a specimen. This is because visible light is more scattered than infrared light in biological tissues [10] and intensity-based detection techniques are less sensitive than interferometric detection techniques [11].

A novel dual modality imaging system has been demonstrated. It is capable of providing simultaneously high resolution FF-OCM images and fluorescence images showing microscopic features of biological tissues. Optical sectioning is achieved in FF-OCM modality owing to the coherence gating property of the light source. In the fluorescence images, it is achieved by using a structured illumination technique. The architecture of the system allows one to obtain full-field images without raster scanning. The two imaging modalities allow one to differentiate structural features in biological samples. The combination of the data types obtained from each imaging modality provides complementary information and could be useful for pathological diagnosis and studying cellular function based on tissue morphology and biochemical properties.

The authors wish to acknowledge the funding agency Réseau Thématique de Recherche Avancée. They are grateful to C. Martin and F. Pain for providing the animal tissues. Institut d'Optique Graduate School is a member of the European Network Photonics4Life.

\section{References}

1. L. P. Hariri, G. T. Bonnema, K. Schmidt, A. M. Winkler, V. Korde, K. D. Hatch, J. R. Davis, M. A. Brewer, and J. K. Barton, Gynecol. Oncol. 114, 188 (2009).

2. J. A. Izatt, M. R. Hee, G. M. Owen, E. A. Swanson, and J. G. Fujimoto, Opt. Lett. 19, 590 (1994).

3. I. Pavlova, M. Williams, A. El-Naggar, R. Richards-Kortum, and A. Gillenwater, Clin. Cancer Res. 14, 2396 (2008).

4. T. J. Muldoon, M. C. Pierce, D. L. Nida, M. D. Williams, A. Gillenwater, and R. Richards-Kortum, Opt. Express 15, 16413 (2007).

5. R. B. Rosen, M. Hathaway, J. Rogers, J. Pedro, P. Garcia, P. Laissue, G. M. Dobre, and A. Gh. Podoleanu, Opt. Express 17, 4112 (2009).

6. S. Yuan, Q. Li, J. Jiang, A. Cable, and Y. Chen, Opt. Lett. 34, 1615 (2009).

7. A. Dubois, K. Grieve, G. Moneron, R. Lecaque, L. Vabre, and C. Boccara, Appl. Opt. 43, 2874 (2004).

8. A. Dubois, G. Moneron, K. Grieve, and A. C. Boccara, Phys. Med. Biol. 49, 1227 (2004).

9. M. A. A. Neil, R. Juskaitis, and T. Wilson, Opt. Lett. 22, 1905 (1997).

10. P. Taroni, A. Pifferi, A. Torricelli, D. Comelli, and R. Cubeddu, Photochem. Photobiol. Sci. 2, 124 (2003)

11. E. Beaurepaire, L. Moreaux, F. Amblard, and J. Mertz, Opt. Lett. 24, 969 (1999). 